\title{
Thermal noise influences fluid flow in thin films during spinodal dewetting
}

\author{
R. Fetzer, ${ }^{1}$ M. Rauscher ${ }^{2}$ R. Seemann,${ }^{3}$ K. Jacobs,${ }^{1}$ and K. Mecke ${ }^{4}$ \\ ${ }^{1}$ Department of Experimental Physics, Saarland University, 66041 Saarbrücken, Germany* \\ ${ }^{2}$ Max-Planck-Institut für Metallforschung, Heisenbergstr. 3, 70569 Stuttgart, Germany \\ ${ }^{3}$ Max-Planck-Institut für Dynamik und Selbstorganisation, Bunsenstr. 10, 37073 Göttingen, Germany \\ 4 Institut für Theoretische Physik, Universität Erlangen-Nürnberg, Staudtstrasse 7, 91058 Erlangen, Germany
}

(Dated: November 9, 2018)

\begin{abstract}
Experiments on dewetting thin polymer films confirm the theoretical prediction that thermal noise can strongly influence characteristic time-scales of fluid flow and cause coarsening of typical length scales. Comparing the experiments with deterministic simulations, we show that the Navier-Stokes equation has to be extended by a conserved bulk noise term to accomplish the observed spectrum of capillary waves. Due to thermal fluctuations the spectrum changes from an exponential to a power law decay for large wavevectors. Also the time evolution of the typical wavevector of unstable perturbations exhibits noise induced coarsening that is absent in deterministic hydrodynamic flow.
\end{abstract}

PACS numbers: 47.61.-k, 47.15.gm, 68.08.Bc, 68.15.+e

With the advent of nanofluidics in the last years it became evident that thermal noise may play an important role in all hydrodynamic processes occuring at free interfaces on small scales. Although in bulk fluids hydrodynamic Navier-Stokes equations are proven to be valid down to the nanometer scale, in free interface flow stochastic forces induced by molecular motion can significantly alter the behavior even on a micrometer scale. Moseler and Landman, for instance, found that the deterministic lubrication approximation for axial-symmetric free boundary flow is not applicable for the description of nanoscopic cylindrical jets [1]. The lack of thermally triggered fluctuations in the classical hydrodynamic continuum modelling was identified as the most likely source for deviations of Navier-Stokes equation from molecular dynamics simulations. They derived a stochastic differential equation that includes thermal noise, whose influence on the dynamics increases as the radius of the nanojet becomes smaller, leading finally to the emergence of symmetric double cone neck shapes during the breakup instead of a long thread solution as expected in the absence of noise. In Ref. [2] path integral methods were applied to confirm that thermal noise induces indeed qualitative changes in the breakup of a liquid nanometer jet: Thermal fluctuations speed up the dynamics and make surface tension an irrelevant force for the breakup. Very recently, the importance of thermal noise for drop formation was observed in a colloidal dispersion with an ultra-low surface tension [3].

Dewetting of thin films is another technologically important free surface flow problem where a lubrication approximation is used widely. So far, thin film flow has been studied by hydrodynamic Navier-Stokes equations without considering thermal noise, e.g., [4]. With decreasing film thickness, however, it is expected that thermal noise gains significance [5]. In Refs. [5, 6, 7], thermal fluctuations have been taken into account and a stochastic version of the thin film equation was derived based on the lubrication approximation for stochastic hydrodynamic equations [8, 9]. The magnitude of the added stochastic forces increases as the thickness of the film decreases. Recent analytical and numerical studies of the stochastic thin film evolution indicate that thermal noise increases characteristic timescales of the dewetting process [6] and changes qualitatively the time evolution of the film thickness $h(\mathbf{r}, t)$ (with $\mathbf{r}=(x, y)$ ), i.e., its power spectrum $\tilde{C}(q, t)[5]$. Here, we report that these predictions of noisy hydrodynamics can be confirmed experimentally by in situ atomic force microscopy (AFM) of dewetting thin polymer films.

For the experiments, we used polystyrene (PS) of $2.05 \mathrm{~kg} / \mathrm{mol}$ molecular weight, prepared on a silicon wafer with a $191 \mathrm{~nm}$ thick silicon oxide layer. By spin casting a toluene solution of PS we prepared films with thicknesses of $(3.9 \pm 0.5) \mathrm{nm}$ and $(4.6 \pm 0.5) \mathrm{nm}$ (as measured by ellipsometry at different sites). These films are unstable and dewet spinodally. Above the glass transition temperature, the PS film is liquid and capillary waves can be observed [10]. Below a characteristic wave number their amplitudes grow exponentially in time, eventually leading to holes in the film [12, 13]. Dewetting then proceeds by the growth of holes and their coalescence. By tapping mode ${ }^{\mathrm{TM}} \mathrm{AFM}$, the entire dewetting process can be monitored in situ, c.f. Fig. 1.

In addition to the experiments, we use deterministic simulation data of the spinodal dewetting process from Ref. [14]. In these simulations, the experimental system parameters film thickness, viscosity, and effective interface potential $\Phi(h)$ were used. We gain quantitative information about the influence of thermal noise to the dewetting process by analyzing the variance $\sigma^{2}(t)=\overline{h^{2}}-\bar{h}^{2}$ of the film height $h(\mathbf{r}, t)$ and the variance $k^{2}(t)=\overline{(\nabla h)^{2}} /\left(2 \pi \sigma^{2}(t)\right)$ of the local slope of $h(\mathbf{r}, t)$. The quantity $k^{2}(t)$ gives information about the preferred wavevector within the film surface in the early stage of dewetting before holes are formed, cf. Fig. 1. In this 


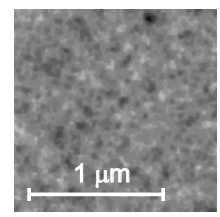

$\mathrm{t}=100 \mathrm{~s}$
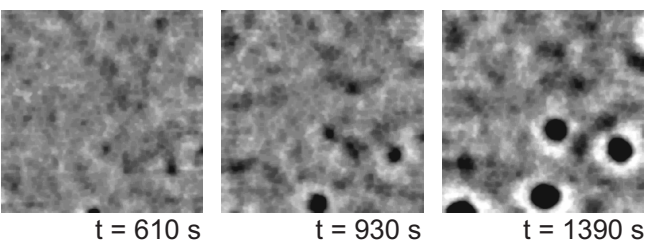

FIG. 1: Dewetting of a $3.9 \mathrm{~nm}$ polystyrene film as monitored by in situ AFM at $53^{\circ} \mathrm{C}$.

early stage regime, the overbars represent an integration over all positions of the image. During the later stage, however, when holes appear and grow, the linearization of the thin film equation (TFEq) [5], which we use to analyse the data, is in general not valid, but may still be used in between the holes, where the film heights are close to the prepared film thickness $h_{0}$. We therefore analyse in the later stage of dewetting the film only at regions where $h(\mathbf{r}, t) \approx h_{0}$ and ignore the other parts. Note that this spatially selective data analysis is not possible by using correlation function or Fourier space techniques.

A main problem in the data analysis is the finite size of the observation window and the emergence of growing holes in the thin film which limits the film regions where linear dynamics can be studied. The variances $\sigma^{2}$ and $k^{2}$ (which are moments of the spectrum) cannot be determined accurately from the Fourier transform of the AFM images. The measured spectrum is convoluted with the Fourier transform of the observation window, which decays as $\sim q^{-2}$ for a sharp cutoff. Thus, the short-wavelength behavior of the spectrum is in our case dominated by the edges of the AFM image and not by the thermal fluctuations. Moreover, the exponentially damped spectrum $\tilde{C} \sim e^{-2 t|\omega(q)|}$, characteristic for the deterministic dynamics, is masked by this convolution yielding a spectrum similar to the algebraic noise-induced $\tilde{C}_{C W}(q) \sim q^{-2}[5]$. Since the 'bare' spectrum $\tilde{C}(q)$ is experimentally not accessible, one has to determine variances $\sigma^{2}$ and $k^{2}$ from the real space images. Whereas determining $\sigma^{2}$ is straightforward, calculating $k^{2}$ from pixelized images using numerical differentiation is too inaccurate.

Here, we apply an analysis technique based on Minkowski functionals $M_{\nu}$ [14]. We threshold the images at a certain film height $h$ and measure the area $M_{0}(h)$, boundary length $M_{1}(h)$, and Euler number $M_{2}(h)$ as function of $h$. In the linear regime of spinodal dewetting, the fluctuations in these quantities should follow a Gaussian distribution which was previously tested in Ref. [14]. Then, the mean values of $M_{\nu}(h)$ depend only on the variances $\sigma^{2}$ and $k^{2}$, which can be determined as fit parameters to the functions $M_{\nu}(h)$. In Fig. 2 we show the resulting temporal evolution of $\sigma^{2}(t)$ and of $k^{2}(t)$ in the linear regime. Besides providing accurate estimations of these values, this morphometric analysis allows to test whether the analysed film region is still in the linear regime. As soon as the non-linear dynamics sets in, the Gaussian model fails to fit the measured functions $M_{\nu}(h)$. We compare the data from two experiments at two spatial resolution lengths (exp. 1: pixel size $r_{p} \approx 6 \mathrm{~nm}$ temperature $T \approx 53^{\circ}$ C; $\exp .2: r_{p} \approx 40 \mathrm{~nm}$, $T \approx 65^{\circ} \mathrm{C}$ ) with data from deterministic simulations at similar physical conditions and find substantial differences. In the following, we explain these differences by introducing thermal noise to the description of thin film flow.

In both experiments the short chain length PS film can be described as an incompressible Newtonian liquid (with a shear viscosity on the order of $\eta \approx 10^{4} \mathrm{Ns} / \mathrm{m}^{2}$ ) on an infinite, flat solid substrate. Thus, the flow can be modelled by a stochastic Navier-Stokes equation with an additional random stress fluctuation tensor representing the effect of thermal molecular motion [8, 9]. We assume no-slip boundary conditions between liquid and substrate and full-slip at the liquid/air interface $z=h(\mathbf{r}, t)$. At the latter, the normal stress is balanced by the surface tension $\gamma$ and the disjoining pressure $-\Phi^{\prime}$. For a smooth thin film, where the characteristic film height $h_{0} \approx 4 \mathrm{~nm}$ is much smaller than the length scale $\approx 100 \mathrm{~nm}$ over which the film thickness varies laterally, a long wavelength expansion yields the following stochastic TFEq [4, [5, 6]

$$
\frac{\partial h}{\partial t}=\nabla\left(\frac{h^{3}}{3 \eta} \nabla\left[\Phi^{\prime}(h)-\gamma \Delta h\right]+\sqrt{\frac{2 k_{B} T h^{3}}{3 \eta}} \mathbf{N}(t)\right)
$$

with a single multiplicative conserved noise vector $\mathbf{N}(\mathbf{r}, t)$ obeying $\langle\mathbf{N}(\mathbf{r}, t)\rangle=0$ and $\left\langle N_{i}(\mathbf{r}, t) N_{j}\left(\mathbf{r}^{\prime}, t^{\prime}\right)\right\rangle=\delta_{i j} \delta(\mathbf{r}-$ $\left.\mathbf{r}^{\prime}\right) \delta\left(t-t^{\prime}\right)$, where $\langle\cdot\rangle$ denotes the ensemble average over realizations of the noise. The characteristic lateral length scale for a thin film of $h_{0}=4 \mathrm{~nm}$ is given by the spinodal wavelength $2 \pi / q_{0}=\sqrt{-8 \pi^{2} \gamma / \Phi^{\prime \prime}\left(h_{0}\right)}=4 h_{0}^{2} \sqrt{\pi^{3} \gamma / A}$, where the Hamaker constant $A$ determines the effective interface potential $\Phi(h)=-\frac{A}{12 \pi h^{2}}$. We have $\frac{2 \pi}{q_{0}} \approx 400 \mathrm{~nm}$ with the values $A \approx 2 \cdot 10^{-20} \mathrm{Nm}$ and $\gamma \approx 3 \cdot 10^{-2} \mathrm{~N} / \mathrm{m}$ determined experimentally for the same system [10]. The experimental parameters lead to dimensionless amplitudes of the noise $\frac{3 k_{B} T}{8 \pi^{2} h_{0}^{2} \gamma} \approx 4 \cdot 10^{-4}$ and $2 \cdot 10^{-4}$ for exps. 1 and 2 , respectively. In the following we show that even this small current induced by thermal fluctuations can substantially influence the dewetting process.

Comparing the experiments with the numerical solution of Eq. (1) with $T=0$, we indeed find excellent agreement of the spatial structures [14]. Nevertheless, the time scales do not match. This can be illustrated by comparing the time dependence of $\sigma^{2}(t)$ and $k^{2}(t)$ for the AFM data and the deterministic thin film simulations, see Fig. 2. The most significant deviation is observed for $k^{2}(t)$ which varies in time for the experiments, but stays constant for the deterministic simulations. This 

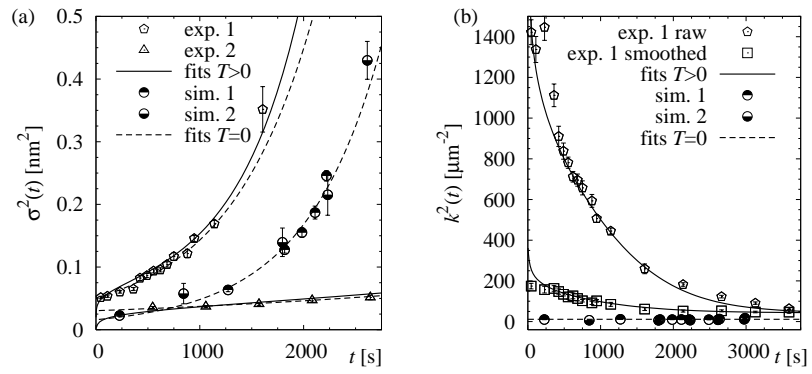

FIG. 2: Analysis of AFM experiments and of deterministic simulations $(T=0)$ : (a) The roughness $\sigma^{2}(t)$ and (b) the variance $k^{2}(t)$ of the local slope (only exp. 1 and simulations for clarity) as functions of time and fits based on the linearized TFEq. While $\sigma^{2}(t)$ can be fitted with the deterministic theory by adjusting the initial roughness $\sigma_{0}^{2}$ and the characteristic time scale $t_{0}$, this is not possible for the experimental $k^{2}(t)$. In the linear regime the deterministic $k^{2}(t)$ is constant in time.

mismatch in $k^{2}(t)$ is most prominent for short times, i.e., for small $\sigma^{2}(t)$. In the following, we quantitatively analyse the early stages of dewetting in a linear approximation of Eq. (11) to demonstrate the relevance of thermal noise in hydrodynamics on small length scales.

In the beginning of the dewetting process the deviations $\delta h(\mathbf{r}, t)=h(\mathbf{r}, t)-h_{0}$ from the initial film height $h_{0}$ are small as compared to $h_{0}$. By expanding Eq. (1) in first order of $\delta h$ and $\mathbf{N}$ we obtain a linear stochastic equation in Fourier space. Its dispersion relation $\omega(q)=[1-$ $\left.\left(q^{2} / q_{0}^{2}-1\right)^{2}\right] / t_{0}$ has its maximum at $q_{0}^{2}=-\Phi^{\prime \prime}\left(h_{0}\right) /(2 \gamma)$ and a characteristic time scale $t_{0}=3 \eta /\left(\gamma h_{0}^{3} q_{0}^{4}\right)$ (about $300 \mathrm{~s}$ and $1700 \mathrm{~s}$ for exp. 1 and 2 , respectively). The spectrum reads $\tilde{C}(q, t)=\left\langle\tilde{\delta} h(\mathbf{q}, t) \tilde{\delta h}^{*}(\mathbf{q}, t)\right\rangle=\tilde{C}_{0}(q) e^{2 \omega(q) t}+$ $\frac{k_{B} T h_{0}^{3}}{3 \eta} \frac{q^{2}}{\omega(q)}\left[e^{2 \omega(q) t}-1\right]$ with the initial spectrum $\tilde{C}_{0}(q)$ at $t=0$. We assume the initial spectrum to be constant $\tilde{C}_{0}(q)=\frac{2 \pi}{q_{0}^{2}} \sigma_{0}^{2}$ for $q<\sqrt{2} q_{0}$ and zero otherwise. The spectrum has necessarily a microscopic cutoff $q_{m}=2 \pi / r_{m} \gg q_{0}$ at the scale $r_{m}$, which is certainly larger than the fluid molecules. The cutoff for the measured spectrum is set by the experimental resolution, e.g., by the pixel size $r_{p}$ or a smoothing length if the data is post-processed. Due to a rapid buildup of noise induced short wavelength roughness on a microscopic time scale $t_{m}=\left(q_{0} / q_{m}\right)^{4} t_{0}$, the short wavelength part of the initial spectrum $\tilde{C}_{0}\left(q>q_{0}\right)$ is irrelevant for the film evolution on the characteristic time scale $t_{0} \gg t_{m}$.

In the case of a spinodally unstable film with $\Phi^{\prime \prime}\left(h_{0}\right)<$ 0 the dispersion relation $\omega(q)$ is negative for $q>\sqrt{2} q_{0}$. This leads to two important differences between deterministic and stochastic dewetting. First, and largely independent of the initial roughness, for $t \rightarrow \infty$ and $q>\sqrt{2} q_{0}$ one finds an exponentially decaying power spectrum $\tilde{C}(q, t) \rightarrow \tilde{C}_{0}(q) e^{-2|\omega(q)| t}$ in the deterministic dynamics $(T=0)$ but we recover the algebraic capillary

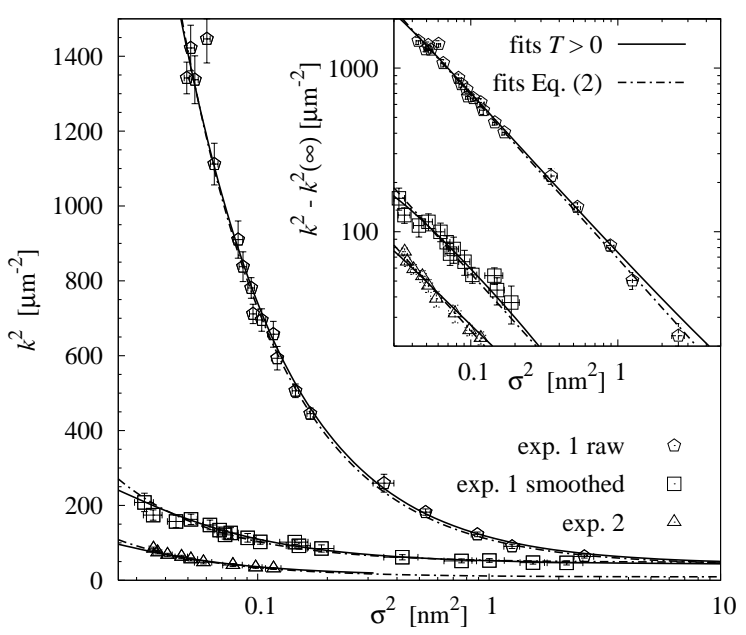

FIG. 3: Slope variance $k^{2}$ from the experiments plotted versus the roughness $\sigma^{2}$. The solid lines are fits based on the lineraized stochastic TFEq, whereas the dash-dotted lines are asymptotic fits according to Eq. (2). The influence of noise is reduced for smoothed as well as for less resolved data (exp.2). The inset shows the same data in a double logarithmic plot highlighting the validity of the asymptotic Eq. (2).

wave spectrum $\tilde{C}(q, t) \rightarrow \frac{k_{B} T h_{0}^{3}}{3 \eta} \frac{q^{2}}{|\omega(q)|} \sim \frac{k_{B} T}{\gamma q^{2}}=\tilde{C}_{C W}(q)$ for any finite temperature $T$. And second, for sufficiently smooth initial spectra which decay rapidly for $q>\sqrt{2} q_{0}$, the maximum of the deterministic spectrum stays at $q_{0}$ for all times, while the maximum of the stochastic spectrum approaches $q_{0}$ from above as $t \rightarrow \infty$, see Ref. [5] for details. This noise generated coarsening process can last until non-linearities become important, effectively masking the most typical feature of the linear deterministic regime, namely that the maximum of the power spectrum stays at the fixed wave number $q_{0}$.

In order to further illustrate the spatio-temporal features of the dynamics we calculate the time evolution of the roughness of the film $\sigma^{2}(t)=\int \frac{d^{2} q}{(2 \pi)^{2}} \tilde{C}(q, t)$ shown in Fig. 2(a) for the experiments discussed above and the corresponding deterministic simulation. Both, the experimental and the deterministic data can be fitted with the deterministic $\sigma_{T=0}^{2}(t)$ with parameters $t_{0}$ and $\sigma_{0}$ in reasonable agreement with the experimental parameters. The initial roughness $\sigma_{0}^{2}$ is experimentally not accessible. Thus, the time evolution of the roughness $\sigma^{2}(t)$ gives no clear indication for the importance of thermal fluctuations for the dewetting process.

Yet, the variance of the local slope $2 \pi \sigma^{2}(t) k^{2}(t)=$ $\left\langle[\boldsymbol{\nabla} \delta h(\mathbf{r}, t)]^{2}\right\rangle=\int \frac{d q q^{3}}{2 \pi} \tilde{C}(q, t)$ is sensitive to thermal noise. We find a strong dependence on the noise term for times $t \sim t_{0}$ whereas the late stage $k^{2}(t \rightarrow \infty)=$ $k_{0}^{2}$ is given by the maximum of the dispersion relation $k_{0}^{2}=q_{0}^{2} /(2 \pi)$ alone. The reason why the noise term becomes irrelevant for large $t$ is the strong increase of $\sigma^{2}(t)$ with time due to the deterministic growth of unstable 
modes. Note that for a purely deterministic dynamics the variance $k_{T=0}^{2}(t)=k_{0}^{2}$ changes in time only at microscopic time scales $t_{m}$ and remains constant in time if one chooses $q_{m}=\sqrt{2} q_{0}$. In particular, the position $q \approx k(t)$ of the maximum in the structure function $\tilde{C}(q, t)$ does not change during a deterministic dewetting process. In contrast, thermal noise induces a time dependence of $k^{2}(t)$. The experimental data cannot be fitted with the deterministic theory, because $k^{2}(t)$ is clearly not constant in time. A simultaneous fit to the experimental data with $\sigma_{0}^{2}=0$ yields $k_{B} T /(4 \pi \gamma)$ and the cut-off wavevector $q_{m}^{2} / q_{0}^{2}$ as given in Table I. From the experimental parameters we would expect $k_{B} T /(4 \pi \gamma) \approx 0.012 \mathrm{~nm}^{2}$ for both experiments, which is reasonably close to the fitted values. The values for $q_{m}$ correspond to cutoff lengths $r_{m}$ which are larger than the nominal pixel size. The reason is probably that the experimental cutoff is smooth rather than sharp. However, the ratios of the $r_{m}$ for the different experiments matches quite well the ratios of the corresponding pixel sizes. In contrast to $\sigma_{0}^{2}$, smoothing changes $k^{2}$ drastically. Deterministic simulation data is hardly affected by smoothing. Smoothing obviously removes fluctuations on small scales, which are exponentially fast damped by the deterministic dynamics but are permanently (re)generated by thermal noise in the experiments. A quantitative analysis of the experiments at different lateral resolution clearly confirms thermal noise as the most likely source for the observed $k^{2}$ dependence. The dependence of $k^{2}$ on the upper cut-off $q_{m}$ is a crucial test of the theoretical prediction as presented below in Eq. (2).

As the microscopic cutoff $q_{m}$ is much larger than $q_{0}$ there is a time regime $t>t_{m} \approx 10^{-2} t_{0}$ up to $t \approx t_{0}$ where $k^{2}(t)$ is approximately given by

$$
k^{2}(t) \approx k_{0}^{2}+\frac{\chi}{\sigma^{2}(t)}, \quad \text { with } \quad \chi=\frac{k_{B} T q_{m}^{2}}{8 \pi^{2} \gamma},
$$

independent of the initial conditions [5]. Since $\sigma^{2}(t)$ is monotonically increasing with time, the characteristic wavelength $\sqrt{2 \pi / k^{2}(t)}$ increases. Thus, thermal noise generates coarsening even in the linear regime for which the deterministic linear dynamics predicts a fixed characteristic wavevector. Fig. 3 shows $k^{2}$ as a function of $\sigma^{2}$ fitted by Eq. (2) as well as by the full linear stochastic theory. Both fits give consistent values for $k_{0}^{2}$ and $\chi$ which are given in Table [ These values are also consistent with the values determined by the system parameters given above.

From the fit values $k_{0}^{2}$ and the theoretical relation $q_{0}^{2}=-\Phi^{\prime \prime}\left(h_{0}\right) /(2 \gamma)$ we get for the film thicknes $h_{0}$ in exp. $13.6 \mathrm{~nm}$ and $3.8 \mathrm{~nm}$ from the smoothed and raw data, respectively, and for exp. $2 h_{0}=5.5 \mathrm{~nm}$. These values are in good agreement with our experimental data. Accordingly, one finds from the fit values for $\chi$ the cut-off lengths $r_{m}$ given in Table \- in good agreement with the ratios of pixels sizes and smoothing length, respectively.
It is remarkable, that one can determine reliable values for film thicknesses and lateral resolution by measuring the effect of thermal noise on fluid flow. This is a strong indication that the coarsening visible in $k^{2}(t)$ during the linear regime is indeed due to thermal fluctuations which can be described by the stochastic TFEq (1). Numerical solutions of Eq. (1) would provide an excellent further test.

In conclusion, the interplay of substrate potentials and thermal noise can strongly influence characteristic time scales of fluid flow and leads to a coarsening of typical lengths on a $\mu \mathrm{m}$ scale. The results will have impact on the future development of nanofluidics, enabling reliable predictions of fluid flow in confined geometries such as in liquid coatings or in lab-on-chip devices.

\begin{tabular}{l|c|c|c|c|c|} 
dataset & $\frac{k_{B} T}{4 \pi \gamma}\left[\mathrm{nm}^{2}\right]$ & $\frac{q_{m}^{2}}{q_{0}^{2}}$ & $k_{0}^{2}\left[\mu \mathrm{m}^{-2}\right]$ & $\chi$ & $r_{m}[\mathrm{~nm}]$ \\
\hline 1 smooth & 0.01 & 12 & 48 & $5.610^{-6}$ & 116 \\
1 raw & 0.01 & 170 & 40 & $710^{-5}$ & 33 \\
2 raw & 0.008 & 28 & 9 & $2.510^{-6}$ & 174
\end{tabular}

TABLE I: Experimental fit parameters for Figs. 2 and 3.

It is a great pleasure to thank J. Becker and G. Grün for granting access to the digital raw data of the simulations. Financial support by the DFG under grants Ja905/3, Se1118/2, Ra1061/2 and Me1361/9 is gratefully acknowledged.

* Present address:Ian Wark Research Institute, University of South Australia, Mawson Lakes, SA 5095, Australia

$\dagger$ Electronic address: mecke@physik.uni-erlangen.de

[1] M. Moseler and Uzi Landman, Science 289, 1165 (2000).

[2] J. Eggers, Phys. Rev. Lett. 89, 084502 (2002).

[3] Y. Hennequin et a., Phys. Rev. Lett. 97, 244502 (2006).

[4] A. Oron, S. H. Davis, and S. G. Bankoff, Rev. Mod. Phys. 69, 931 (1997).

[5] K. Mecke and M. Rauscher, J. Phys.: Condens. Matter 17, S3515 (2005).

[6] G. Grün, K. Mecke and M. Rauscher, J. Stat. Phys. 122, 1261 (2006)

[7] B. Davidovitch, E. Moro, and H. A. Stone, Phys. Rev. Lett. 95, 244505 (2005).

[8] L. D. Landau and E. M. Lifšic, Statistische Physik, 5th ed. (Akademie Verlag, 1991).

[9] R. F. Fox and G. E. Uhlenbeck, Phys. Fluids 13, 1893 (1970).

[10] R. Seemann, S. Herminghaus, and K. Jacobs, Phys. Rev. Lett. 86, 5534 (2001); J. Phys.: Condens. Matter 13, 4925 (2001).

[11] A. Vrij, Discuss. Faraday Soc. 42, 23 (1966).

[12] E. Ruckenstein and R. K. Jain, J. Chem. Soc. Faraday Trans. II, 132 (1974).

[13] S. Herminghaus et al., Science 282, 916 (1998).

[14] J. Becker, G. Grün, R. Seemann, H. Mantz, K. Jacobs, K. Mecke, and R. Blossey, Nature Materials 2, 59 (2003). 